\title{
Isometric Reflection Vectors and Characterizations of Hilbert Spaces
}

\author{
Donghai Ji and Senlin Wu \\ Department of Applied Mathematics, Harbin University of Science and Technology, Harbin 150080, China \\ Correspondence should be addressed to Senlin Wu; wusenlin@outlook.com
}

Received 31 December 2013; Accepted 18 February 2014; Published 18 March 2014

Academic Editor: Yongqiang Fu

Copyright (C) 2014 D. Ji and S. Wu. This is an open access article distributed under the Creative Commons Attribution License, which permits unrestricted use, distribution, and reproduction in any medium, provided the original work is properly cited.

\begin{abstract}
A known characterization of Hilbert spaces via isometric reflection vectors is based on the following implication: if the set of isometric reflection vectors in the unit sphere $S_{X}$ of a Banach space $X$ has nonempty interior in $S_{X}$, then $X$ is a Hilbert space. Applying a recent result based on well-known theorem of Kronecker from number theory, we improve this by substantial reduction of the set of isometric reflection vectors needed in the hypothesis.
\end{abstract}

\section{Introduction}

Throughout this paper $X=(X,\|\cdot\|)$ always denotes a real Banach space with origin $o$, unit ball $B_{X}$, and unit sphere $S_{X}$. The dimension $\operatorname{dim} X$ of such a space is always assumed to be at least 2 . When $\operatorname{dim} X=2, X$ is called a Minkowski plane. For two linearly independent vectors $x$ and $y$ in $X$, we denote by $L(x, y)$ the Minkowski plane spanned by $x$ and $y$.

Following [1], a closed linear subspace $M$ of $X$ is said to be an isometric reflection subspace of $X$ if there exists a linear subspace $N$ of $X$ such that $X=M \oplus N$ and that $\|x+y\|=$ $\|x-y\|$ holds (i.e., $x$ is isosceles orthogonal to $y$; see, e.g., [2]) for every $x \in M$ and every $y \in N$. In this situation the linear map $R_{M}: X \mapsto X$ defined by $R_{M}(x+y)=x-y$, where $x \in M$ and $y \in N$, is a surjective linear isometry and is called the isometric reflection of $M$ in $X$. In particular, if an isometric reflection subspace $M$ is spanned by a unit vector $u$, then such a unit vector is called an isometric reflection vector. In this case the isometric reflection $R_{M}$ is simply denoted by $R_{u}$. The set of isometric reflection vectors in $S_{X}$ is denoted by $I_{X}^{r}$. The notion of isometric reflection vector is closely related to another type of orthogonality in normed linear spaces: a vector $x \in X$ is said to be Roberts orthogonal to another vector $y$ (denoted by $\left.x \perp_{R} y\right)$ if $\|x+\alpha y\|=\|x-\alpha y\|$ holds for each real number $\alpha$. It is not difficult to verify the following lemma.

Lemma 1 (cf. $[3,4])$. A unit vector $u$ lies in $I_{X}^{r}$ if and only if there exists a hyperplane $H_{u}$ passing through the origin o such that $u \perp_{R} H_{u}$. Moreover, if $u$ lies in $I_{X}^{r}$, then the hyperplane $H_{u}$ is uniquely determined.

For more information concerning geometric properties of isometric reflection vectors we refer to the recent papers $[3,5,6]$.

Becerra Guerrero and Rodriguez Palacios [4] proved the following interesting characterization of Hilbert spaces (see [1] for a different proof).

Theorem 2 (cf. $[1,4])$. If $I_{X}^{r}$ has nonempty relative interior in $S_{X}$, then $X$ is a Hilbert space.

Our aim is to improve Theorem 2. Before stating our main result, we still need some definitions and notation about arc lengths.

Let $u$ and $v$ be two unit vectors in a Minkowski plane $X$. If $u \neq-v$, then the set

$$
\{\alpha u+\beta v: \alpha, \beta \geq 0\} \cap S_{X}
$$

is called the (minor) arc connecting $u$ to $v$ and denoted by $\operatorname{arc}(u, v)$. The length of $\operatorname{arc}(u, v)$ is denoted by $\delta_{X}(u, v)$. If $u=-v$ then $\delta_{X}(u, v)$ is set to be $\left|S_{X}\right| / 2$, where $\left|S_{X}\right|$ is the circumference of $S_{X}$. We will also make use of directed arc length. Fix an orientation $\omega$ on $X$. Let $u$ and $v$ be two distinct points in $S_{X}$. The part of $S_{X}$ connecting $u$ to $v$ in the positive orientation is called the directed arc connecting $u$ to $v$, and its length is called the directed arc length from $u$ to $v$ and denoted 
by $\overrightarrow{\delta_{X}}(u, v)$. See $[7$, p. 112] or the survey paper [8] for the definitions of arc length and circumference. For an arbitrary number $\alpha \in(0,1)$ we denote by $A_{\alpha}(u)$ and $A_{\alpha}^{-1}(u)$ the two points in $S_{X}$ such that

$$
\overrightarrow{\delta_{X}}\left(A_{\alpha}^{-1}(u), u\right)=\overrightarrow{\delta_{X}}\left(u, A_{\alpha}(u)\right)=\alpha\left|S_{X}\right| .
$$

Moreover, we define $A_{\alpha}^{0}(u):=u$ and, for an integer $n \geq 1$,

$$
A_{\alpha}^{n}(u):=A_{\alpha}\left(A_{\alpha}^{n-1}(u)\right), \quad A_{\alpha}^{-n}(u):=A_{\alpha}^{-1}\left(A_{\alpha}^{-n+1}(u)\right) .
$$

The following theorem is our main result.

Theorem 3. If there exists a vector $u \in I_{X}^{r}$ such that each twodimensional subspace $L$ of $X$ containing $u$ contains a vector $v \in$ $I_{X}^{r}$ such that $\delta_{X}(u, v) /\left|S_{L(u, v)}\right|$ is irrational and less than $1 / 4$, then $X$ is a Hilbert space.

Compared with Theorem 2, the cardinality of the set of isometric reflection vectors involved in the hypothesis of Theorem 3 is substantially reduced.

\section{Proof of Theorem 3}

The following result is well known in number theory.

Lemma 4 (Kronecker's Theorem, cf. [9, Theorem 439, p. 376]). If $\alpha$ is an irrational number, then the set of all numbers of the form

$$
\{n \alpha\}:=n \alpha-[n \alpha]
$$

where $[n \alpha]$ is the largest integer which does not exceed $n \alpha$, is dense in $(0,1)$.

Based on the above lemma, Martini and $\mathrm{Wu}[10]$ proved the following lemma, which is our main tool.

Lemma 5 (cf. [10]). Let $X$ be a Minkowski plane. Then, for any point $u \in S_{X}$ and any irrational number $\alpha \in(0,1)$, the set $\left\{A_{\alpha}^{n}(u)\right\}_{n=-\infty}^{+\infty}$ is dense in $S_{X}$.

Lemma 6. Let $u$ be a unit vector in $I_{X}^{r}$ and $T$ be a linear isometry on $X$. Then $T(u) \in I_{X}^{r}$.

Proof. Since $u \in I_{X}^{r}$, there exists a hyperplane $H_{u}$ passing through the origin and satisfying $u \perp_{R} H_{u}$. It is clear that $T\left(H_{u}\right)$ is also a hyperplane passing through the origin. For each vector $T(v) \in T\left(H_{u}\right)$ and each number $\alpha \in \mathbb{R}$, we have the following equalities:

$$
\|T(u)+\alpha T(v)\|=\|u+\alpha v\|=\|u-\alpha v\|=\|T(u)-\alpha T(v)\| .
$$

This implies that $T(u) \perp_{R} T\left(H_{u}\right)$. Thus $T(u) \in I_{X}^{r}$.

Before proving Theorem 3 we list some elementary properties of the isometric reflection.
Lemma 7. Let $u \in S_{X}$ be an isometric reflection vector. Then the following statements hold true.

(1) $R_{u}\left(R_{u}(x)\right)=x$ holds for each $x \in X$.

(2) For each $x \in X$, the midpoint of $x$ and $R_{u}(x)$ is contained in the one-dimensional space spanned by $u$ and the difference between them lies in $H_{u}$.

(3) For two unit vectors $x, y$ satisfying $x \neq-y, R_{u}$ maps arc $(x, y)$ to $\operatorname{arc}\left(R_{u}(x), R_{u}(y)\right)$ preserving arc length.

(4) For each vector $v$ in $H_{u}$,

$$
\delta_{X}(u, v)=\frac{1}{4}\left|S_{L(u, v)}\right|
$$

(5) The image of $I_{X}^{r}$ under $R_{u}$ is contained in $I_{X}^{r}$.

Proof. By Lemma 1, there exists a unique hyperplane $H_{u}$ passing through the origin $o$ such that $u \perp_{R} H_{u}$. Then, for each point $x \in X$, there exist a unique number $\alpha(x)$ and a unique point $x_{H} \in H_{u}$ such that

$$
x=\alpha(x) u+x_{H} .
$$

It is clear that $R_{u}(x)=\alpha(x) u-x_{H}$. Thus (1) and (2) are both true. (3) follows directly from the fact that $R_{u}$ is a linear isometry. (5) is a direct corollary of Lemma 6. It remains to prove (4).

Clearly,

$$
\begin{aligned}
& S_{L(u, v)} \\
& \quad=\operatorname{arc}(u, v) \cup \operatorname{arc}(u,-v) \cup \operatorname{arc}(-u,-v) \cup \operatorname{arc}(-u, v) .
\end{aligned}
$$

Moreover, (3) implies that $\delta_{X}(u, v)=\delta_{X}(u,-v)$, from which (4) follows.

The following lemma is a direct corollary of $\left(3.3^{\prime}\right)$ in [11], and we omit the proof.

Lemma 8. Let $X$ be a Banach space. If $I_{X}^{r}=S_{X}$, then $X$ is a Hilbert space.

Proof of Theorem 3. We only need to show that, under the hypothesis of the theorem, each unit vector $x$ is an isometric reflection vector.

We may assume that $x$ and $u$ are linearly independent, since otherwise there is nothing to prove. Let $L=L(u, x)$ and fix an orientation $\omega$ on $L$. Denote by $u_{H}$ the point in $S_{L} \cap H_{u}$ such that the orientation from $u$ to $u_{H}$ is $\omega$. By the hypothesis of the theorem, there exists an isometric reflection vector $v \in L$ such that $\delta_{X}(u, v)$ is irrational and less than $(1 / 4)\left|S_{L}\right|$. Suppose that $v=\gamma u+\eta u_{H}$, where $\gamma$ and $\eta$ are two real numbers. Then $\gamma>0$. Replacing $v$ by $R_{u}(v)$, which is also an isometric reflection vector, we may, if necessary, also require that $\eta>0$. Then $v \in \operatorname{arc}\left(u, u_{H}\right)$.

Put

$$
\alpha=\frac{\delta_{X}(u, v)}{\left|S_{L}\right|}, \quad x^{0}:=A_{\alpha}^{0}(u)=u \text {. }
$$


Then, since $v$ is the unique vector in $S_{L}$ such that $\overrightarrow{\delta_{X}}(u, v)=$ $\alpha\left|S_{L}\right|$, we have

$$
x^{1}:=A_{\alpha}(u)=v \text {. }
$$

Since $R_{u}$ maps $\operatorname{arc}(u, v)$ to $\operatorname{arc}\left(u, R_{u}(v)\right)$ and preserves arc length, we have

$$
x^{-1}:=A_{\alpha}^{-1}(u)=R_{u}(v)=R_{x_{0}}\left(x^{1}\right) .
$$

Thus $x^{0}, x^{1}$, and $x^{-1}$ are all isometric reflection vectors. We continue by setting

$$
\begin{aligned}
x^{2} & :=A_{\alpha}(v)=A_{\alpha}^{2}(u), \\
x^{-2} & :=A_{\alpha}^{-1}\left(x^{-1}\right)=A_{\alpha}^{-2}(u) .
\end{aligned}
$$

On the one hand, since $R_{v}$ maps $\operatorname{arc}(u, v)$ to $\operatorname{arc}\left(v, R_{v}(u)\right)$,

$$
x^{2}=R_{v}(u)=R_{x^{1}}\left(x^{0}\right) .
$$

Similarly,

$$
x^{-2}=R_{x^{-1}}(u)=R_{x^{-1}}\left(x^{0}\right) .
$$

Now $x^{2}$ and $x^{-2}$ are both isometric reflection vectors of $X$. By repeating such constructions, we can obtain a set of isometric reflection vectors $\left\{x^{n}: n \in \mathbb{Z}\right\}$ of $X$ in $L$ such that, for each $n \geq 2$,

$$
\begin{aligned}
x^{n} & =A_{\alpha}^{n}(u)=R_{x^{n-1}}\left(x^{n-2}\right), \\
x^{-n} & =A_{\alpha}^{-n}(u)=R_{x^{-n+1}}\left(x^{-n+2}\right) .
\end{aligned}
$$

From Lemma 5 it follows that the set $\left\{x^{n}: n \in \mathbb{Z}\right\}$ is dense in $S_{L}$. Thus $x$ lies in the closure of $\left\{x^{n}: n \in \mathbb{Z}\right\}$. Since the set of isometric reflection vectors in $X$ is closed, this implies that $x$ itself is an isometric reflection vector. This completes the proof.

\section{Conflict of Interests}

The authors declare that there is no conflict of interests regarding the publications of this paper.

\section{Acknowledgments}

The corresponding author is partially supported by a 973 program (Grant no. 2013CB834201) and both of the authors are supported by a foundation from the Ministry of Education of Heilongjiang Province (Grant no. 1251H013), the Nature Science Foundation of Heilongjiang Province (Grant no. A201011), the National Nature Science Foundation of China (Grant nos. 11371114 and 11171082), China Postdoctoral Science Foundation (Grant nos. 2012M520097 and 2013T60019), and by the Scientific Research Foundation for the Returned Overseas Chinese Scholars, State Education Ministry.

\section{References}

[1] A. Aizpuru, F. J. García-Pacheco, and F. Rambla, "Isometric reflection vectors in Banach spaces," Journal of Mathematical Analysis and Applications, vol. 299, no. 1, pp. 40-48, 2004.

[2] J. Alonso, H. Martini, and S. Wu, "On Birkhoff orthogonality and isosceles orthogonality in normed linear spaces," Aequationes Mathematicae, vol. 83, no. 1-2, pp. 153-189, 2012.

[3] C. He, H. Martini, and S. Wu, "On bisectors for convex distance functions," Extracta Mathematicae, vol. 28, no. 1, pp. 57-76, 2013.

[4] J. Becerra Guerrero and A. Rodriguez Palacios, "Isometric reflections on Banach spaces after a paper of A. Skorik and M. Zaidenberg," Rocky Mountain Journal of Mathematics, vol. 30, no. 1, pp. 63-83, 2000.

[5] F. J. García-Pacheco, "Geometry of isometric reflection vectors," Mathematica Slovaca, vol. 61, no. 5, pp. 807-816, 2011.

[6] C. Hao and S. Wu, "Homogeneity of isosceles orthogonality and related inequalities," Journal of Inequalities and Applications, vol. 2011, article 84, 2011.

[7] A. C. Thompson, Minkowski Geometry, vol. 63 of Encyclopedia of Mathematics and its Applications, Cambridge University Press, Cambridge, UK, 1996.

[8] H. Martini, K. J. Swanepoel, and G. Weiß, "The geometry of Minkowski spaces-a survey. I," Expositiones Mathematicae, vol. 19, no. 2, pp. 97-142, 2001.

[9] G. H. Hardy and E. M. Wright, An Introduction to the Theory of Numbers, Oxford University Press, New York, NY, USA, 5th edition, 1979.

[10] H. Martini and S. Wu, "Halving circular arcs in normed planes," Periodica Mathematica Hungarica, vol. 57, no. 2, pp. 207-215, 2008.

[11] D. Amir, Characterizations of Inner Product Spaces, vol. 20 of Operator Theory: Advances and Applications, Birkhäuser, Basel, Switzerland, 1986. 


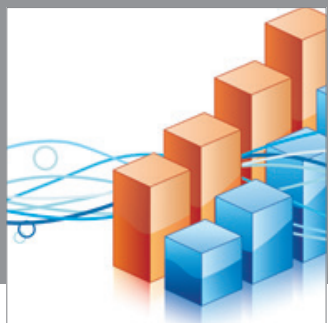

Advances in

Operations Research

mansans

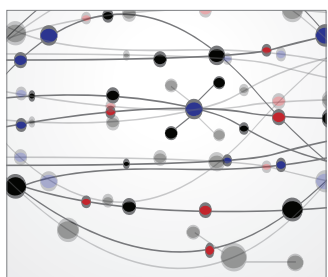

The Scientific World Journal
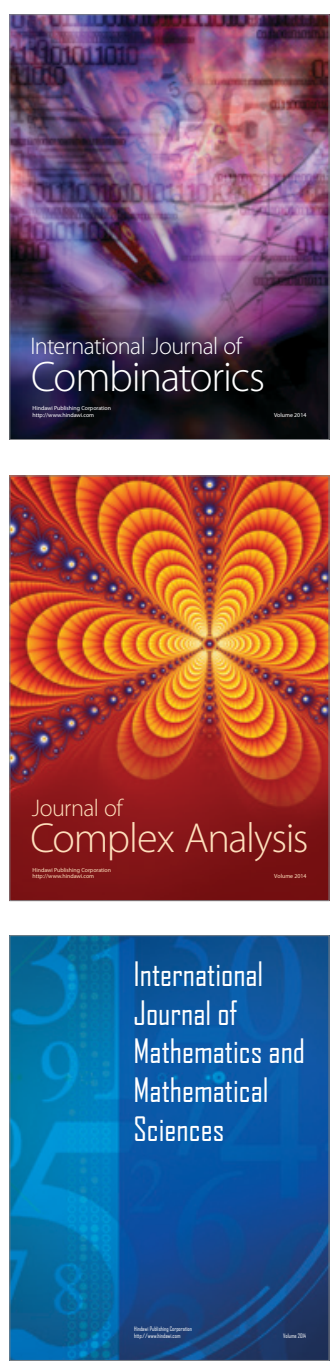
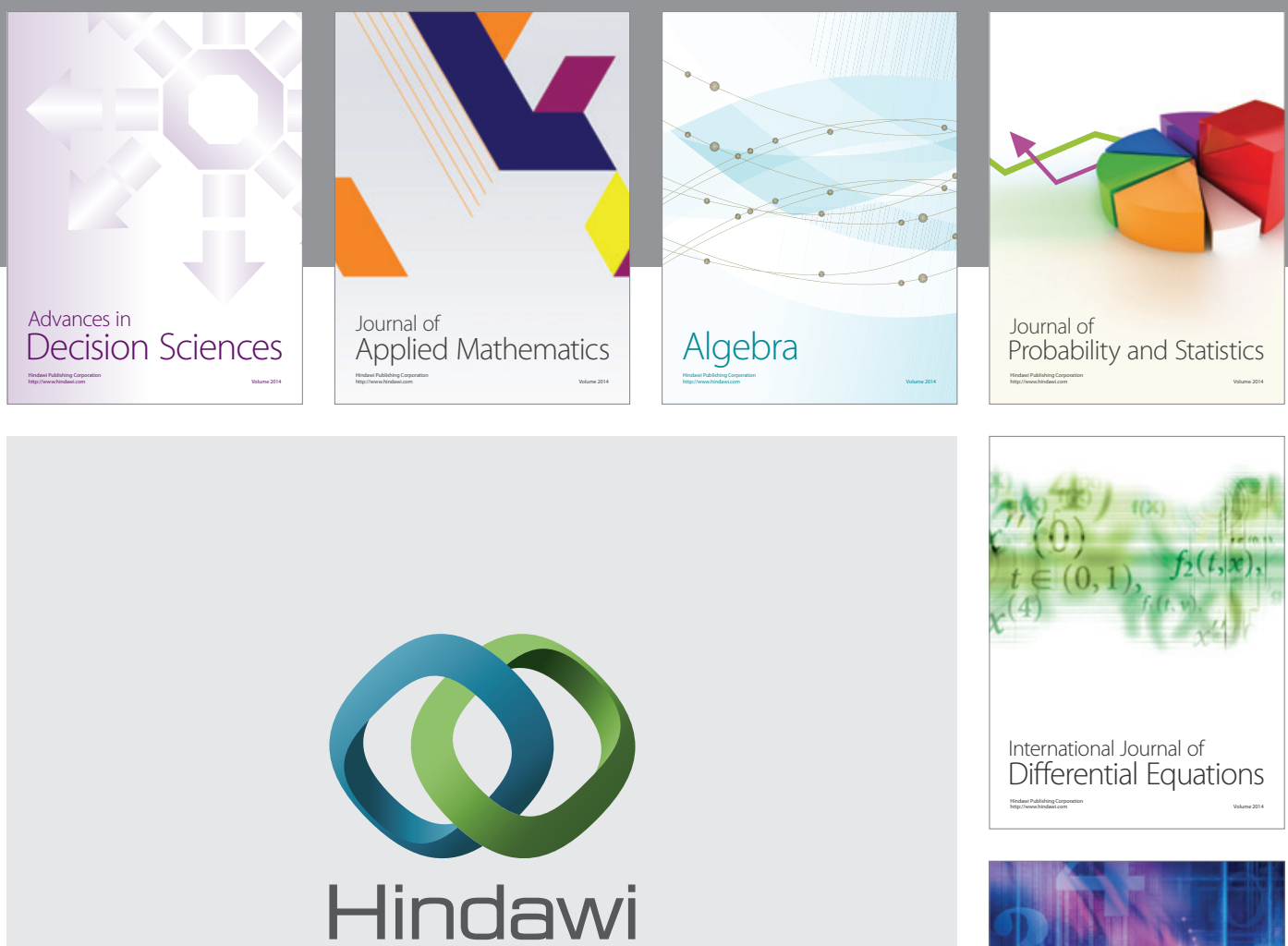

Submit your manuscripts at http://www.hindawi.com
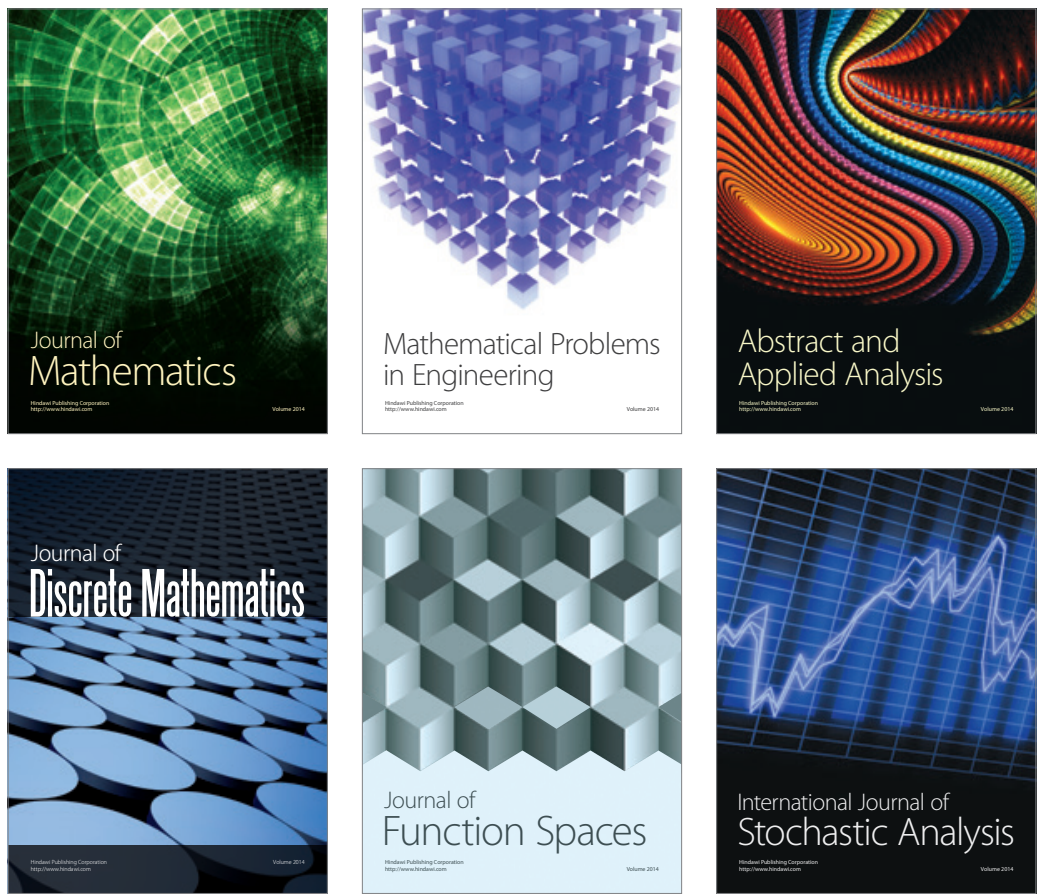

Journal of

Function Spaces

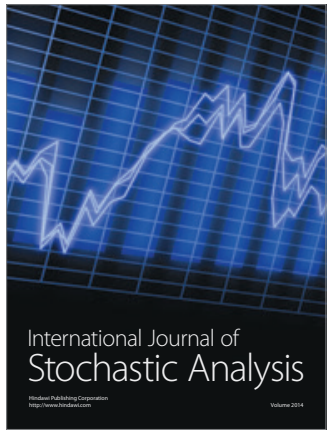

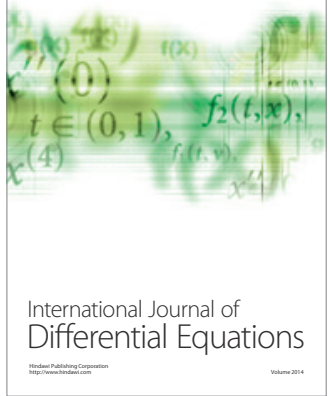
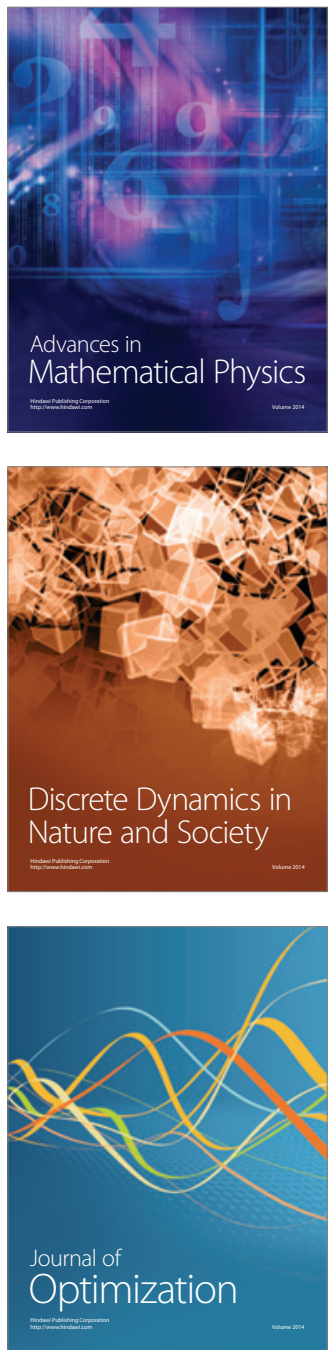